\title{
Bias-induced modulation of ultrafast carrier dynamics in metallic single-walled carbon nanotubes
}

\author{
Keisuke Maekawa, ${ }^{1}$ Kazuhiro Yanagi, ${ }^{2}$ Yasuo Minami, ${ }^{1,3}$ Masahiro Kitajima,,${ }^{1,4}$ Ikufumi Katayama, ${ }^{1, *}$ and Jun Takeda ${ }^{1}$ \\ ${ }^{1}$ Department of Physics, Graduate School of Engineering, Yokohama National University, Tokiwadai 79-5, Yokohama 240-8501, Japan \\ ${ }^{2}$ Department of Physics, Graduate School of Science and Engineering, Tokyo Metropolitan University, \\ Minami-Osawa 1-1, Hachioji 192-0397, Japan \\ ${ }^{3}$ Institute of Technology and Science, Graduate School of Advanced Technology and Science, Tokushima University, \\ Minamijou-Sanjimacho 2-1, Tokushima 770-8506, Japan \\ ${ }^{4}$ LxRay Co. Ltd., Koshienguchi 3-28-22, Nishinomiya 663-8172, Japan \\ ${ }^{5}$ Department of Applied Physics, National Defense Academy, Hashirimizu 1- 10-20, Yokosuka 239-8686, Japan
}

(Received 16 August 2017; revised manuscript received 20 December 2017; published 22 February 2018)

\begin{abstract}
The gate bias dependence of excited-state relaxation dynamics in metallic single-walled carbon nanotubes (MCNTs) was investigated using pump-probe transient absorption spectroscopy coupled with electrochemical doping through an ionic liquid. The transient transmittance decayed exponentially with the pump-probe delay time, whose value could be tuned via the Fermi-level modulation of Dirac electrons under a bias voltage. The obtained relaxation time was the shortest when the Fermi level was at the Dirac point of the MCNTs, and exhibited a U-shaped dependence on the bias voltage. Because optical dipole transitions between the Dirac bands are forbidden in MCNTs, the observed dynamics were attributed to carrier relaxation from the $E_{11}$ band to the Dirac band. Using a model that considers the suppression of electron-electron scattering (impact ionization) due to Pauli blocking, we could qualitatively explain the obtained bias dependence of the relaxation time.
\end{abstract}

DOI: 10.1103/PhysRevB.97.075435

\section{INTRODUCTION}

Carbon-based nanoscale materials such as carbon nanotubes (CNTs) and graphene have been intensively studied as they display intriguing physical, electrical, and optical properties, such as high mobility and high thermal conductivity [1-6]. In particular, in CNTs, the electronic states can easily be controlled by a slight change of the structure, so-called chirality, from semiconductors to metals. In metallic CNTs (MCNTs), electronic conduction occurs via the quasi-onedimensional Dirac electrons with a linear energy-momentum dispersion curve.

In MCNTs, a band-to-band transition with a large oscillator strength ( $E_{11}$ band) is observed in the visible wavelength range [7]. Upon photoexcitation of MCNTs, both the excited $E_{11}$ band and Dirac electrons are expected to contribute to unique carrier-carrier interactions. However, the ultrafast dynamics of the photoexcited carriers in MCNTs have not been as extensively studied as those of semiconducting CNTs [8-13], partly because it has been difficult to extract pure MCNTs until recently [14].

One powerful way of investigating the carrier dynamics in metals and semimetals is to perform time-resolved spectroscopy while tuning the Fermi level $[15,16]$. In MCNTs, tuning the Fermi level over several eV allows optoelectric properties to be analyzed, for example, drastic color changes have been reported [17-20]. These results indicate that the Fermi energy or the carrier concentration of the Dirac band can be controlled by the bias voltage; therefore, we expect to

\footnotetext{
*katayama@ynu.ac.jp
}

elucidate the mechanism of the coupling dynamics between Dirac and band electrons in the MCNTs. As carrier-carrier interactions are of fundamental importance in optical and electrical applications, we investigated the dynamics of Dirac and band electrons in MCNTs using sub-10-fs pump-probe spectroscopy via electrochemical doping by applying a gate voltage through an ionic liquid.

\section{EXPERIMENT}

In the experiments, we used MCNTs with the average diameter of $1.4 \mathrm{~nm}$, which were purified by the density-gradient method [21]. The purity of the MCNTs was estimated to be greater than 95\% [17]. MCNT thin films were formed by vacuum filtration and were placed on glass slides with $\mathrm{Ti}$ $(5 \mathrm{~nm}) / \mathrm{Au}(100 \mathrm{~nm})$ electrodes. The orientations of the MCNTs were thus random in our sample. To apply a voltage to these samples, a droplet of an ionic liquid, TMPA-TFSI $(N, N, N$ trimethyl- $N$-propylammonium bis(trifluoromethanesulfonyl) imide; Kanto-Kagaku Co.) was placed on the sample. Figure 1(a) shows a schematic of the sample configuration, in which the polarity of the bias voltage determines the carrier type (electrons or holes) to be injected. To estimate the actual bias voltage on the sample, we measured the probe voltage using the effective circuit shown in the figure. The probe voltage as a function of applied voltage is shown in Fig. 1(b). The MCNT sample used in this study reached the chargeneutral point or the Dirac point around the applied voltage of $1.0 \mathrm{~V}$, where the Fermi level of the MCNTs could be tuned to the Dirac point. We confirmed that none of MCNT samples were damaged after the experiments. All of the experiments were performed at room temperature of about $293 \mathrm{~K}$. 


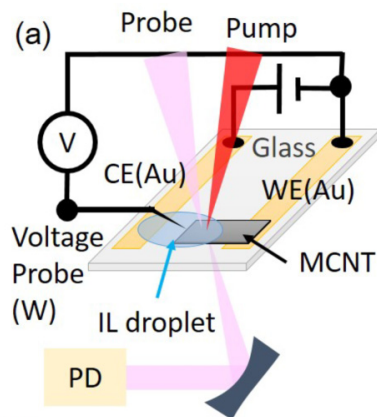

(b)

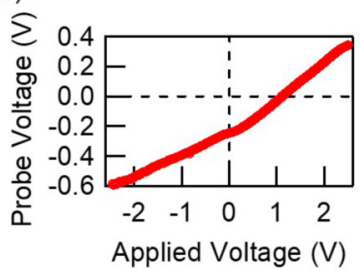

(c)

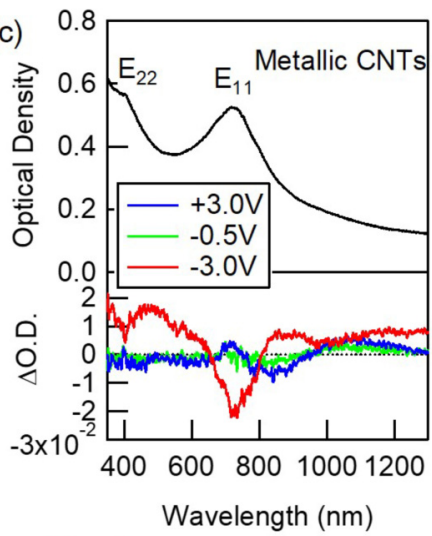

(d)

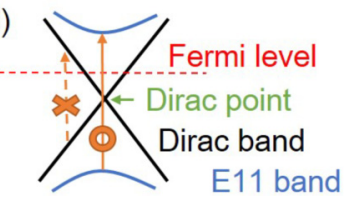

FIG. 1. (a) Schematic of the experimental configuration for pump-probe spectroscopy under a bias voltage. The probe voltage was measured using a tungsten pin near the MCNT sample deposited on a glass plate. CE: counter electrode; IL: ionic liquid droplet; PD: photodiode. (b) Probe voltage as a function of the applied voltage. (c) Absorption spectrum (optical density) of the MCNT thin film. The lower panel shows the difference of the absorption under the bias voltage. (d) Schematic of the excitation processes in the MCNTs near the Dirac point. The vertical arrows indicate the allowed (solid line) and forbidden (dashed line) transitions between the valence and conduction band.

Figure 1(c) shows the absorbance spectrum of the MCNT film. Two absorbance peaks due to the excitonic resonance (or van Hove singularity) were observed at $720 \mathrm{~nm}\left(E_{11}\right)$ and $400 \mathrm{~nm}\left(E_{22}\right)$. When applying a bias voltage to the sample, we observed a slight change in the shape of the absorption spectrum, as plotted for a few different voltages in the lower panel of Fig. 1(c). Under negative bias voltages, the $E_{11}$ absorption bleached and broadband absorption was induced in the observed wavelength range. Because the charge-neutral point is located around a positive bias of $1.0 \mathrm{~V}$, the data under a negative bias voltage of $-3.0 \mathrm{~V}$ only shows the bleaching due to the Pauli blocking of the $E_{11}$ transition around $700 \mathrm{~nm}$ as well as the bias-induced broad absorption around $850 \mathrm{~nm}$ probably due to the plasmon absorption of the $E_{11}$ band electrons [15]. On the other hand, the bias of $+3.0 \mathrm{~V}$ could not yield the bleaching because the actual Fermi energy of the sample was insufficient to induce it. Note that the band-to-band transition, depicted by the dashed line in Fig. 1(d), is forbidden by the symmetry [22]. In addition, the collective response of the Dirac electrons existed at a much lower frequency [23], and may have little dependence on the Fermi energy because of the constant density of states and Fermi velocity of the Dirac band in one dimension.

To observe the carrier lifetime in MCNT samples under a bias voltage, we used a degenerate pump-probe spectroscopy setup with sub-10-fs time resolution. A Ti:sapphire laser system (repetition rate: $80 \mathrm{MHz}$; pulse duration: $7.5 \mathrm{fs}$; wavelength range: $680-980 \mathrm{~nm} ; 300 \mathrm{~mW}$ ) was employed for this study.
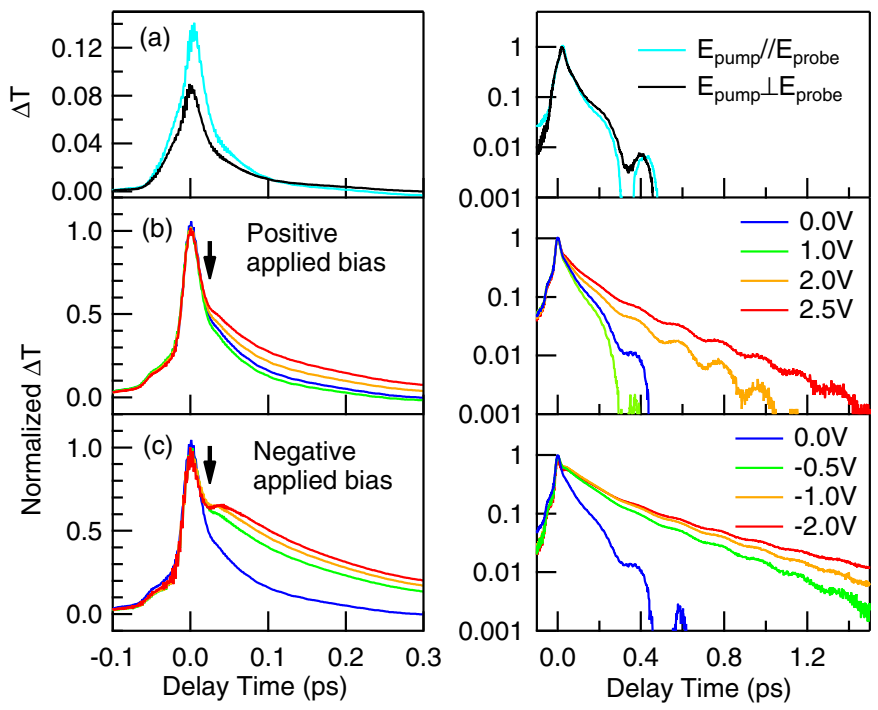

FIG. 2. (a) Pump-probe transient transmittance change observed with the polarization configuration parallel and perpendicular to each other. (b) Normalized transient transmittance under a positive bias voltage with the parallel polarization configuration. (c) Normalized transient transmittance under the negative bias voltage with the parallel polarization configuration. The right panel shows the logarithmic plot, while the left panel shows the linear plot near the time origin. The baselines for the data in the logarithmic plots are offset to clearly show the exponential decay. The arrows indicate the onset of nonexponential decay near the time origin observed as a kink structure.

The wavelength range of this laser system covered the $E_{11}$ transition and the plasmonic absorption band regions. We measured both configurations where polarizations of the pump and probe beams were parallel and perpendicular to each other. The average power of the pump pulse was $100 \mathrm{~mW}$, while that of the probe was $5 \mathrm{~mW}$. The diameter of the focusing area at the sample was around $50 \mu \mathrm{m}$.

\section{EXPERIMENTAL RESULTS}

Figure 2(a) shows the polarization dependence of the transient absorption observed at a bias voltage of $0 \mathrm{~V}$. Because of the anisotropy of the absorption coefficient in MCNTs, the different polarization configuration gave rise to a slight difference in the peak intensity. However, the dynamic behavior, such as carrier relaxation, was almost the same for both parallel and perpendicular polarizations, as reported in a previous study for semiconducting CNTs [24]. In order to clearly elucidate the carrier dynamics, the time evolution of the transient transmittance is plotted as a semilog graph for parallel polarization, as shown in the left panel of Fig. 2. Here, we note that the observed decay dynamics were independent of the pump fluence under our experimental conditions.

At around $30 \mathrm{fs}$, we observed a kink structure, which could be assigned to the dynamics from initial nonequilibrium electron distribution to the thermal equilibrium. After the thermalization was completed, the transmittance showed a single-exponential decay together with the coherent phonon oscillations of the radial breathing mode. When the bias voltage 


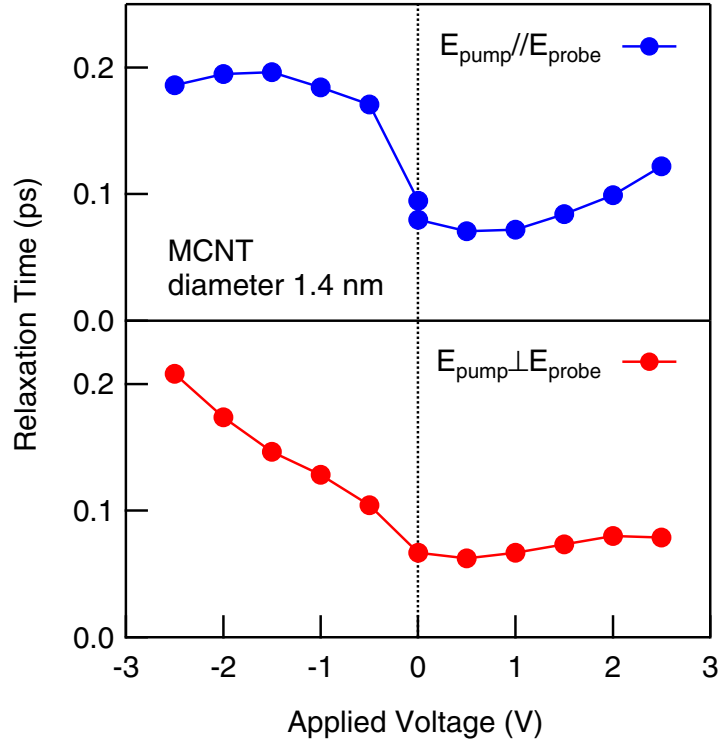

FIG. 3. Relaxation time as a function of bias obtained by the exponential fitting for parallel (upper panel) and perpendicular (lower panel) polarization configurations.

was applied, the transient absorption changed drastically, as shown in Figs. 2(b) and 2(c). The rate of decay was slower in the case of electron doping (negative polarity) than that with a positive bias voltage, and was the shortest with a bias voltage of $0.5-1.0 \mathrm{~V}$; this was due to the shifted neutral point observed in Fig. 1(b). These results demonstrate that the carrier relaxation process was accelerated at the charge-neutral point. It is worth noting that the range of probe bias voltage $(-0.6-0.4 \mathrm{eV})$ observed in Fig. 1(b) was far from the energy where the band-to-band transition is fully bleached as shown in Fig. 1(c); therefore, the change in the relaxation dynamics as a function of bias voltage should be due to interactions with the Dirac electrons. Note that the observed decay dynamics are independent of the pump fluence in our experimental conditions.

To clarify the origin of the change in transient transmittance, the obtained carrier relaxation times are plotted as a function of a bias voltage in Fig. 3. Overall, we observed a minimum carrier relaxation time at around $0.5-1.0 \mathrm{~V}$, which may indicate an intrinsic relaxation at the Dirac point of MCNTs. We also observed that the relaxation time increased more than a factor of 2 from the fastest. This fact shows that the carrier relaxation time can be controlled by the bias voltage. The overall tendency of the relaxation times as a function of bias voltage was similar for both polarizations. In the following section, we will consider the origin of the relaxation behavior with a simple model considering the Coulomb interaction between the $E_{11}$ band and Dirac electrons, i.e., impact ionization, and the electron-phonon scattering processes.

\section{DISCUSSION}

The impact ionization process is one of the dominant relaxation paths for excited electrons in graphene and other materials $[13,25,26]$. Because the dipole transition between the Dirac states is forbidden in MCNTs, as shown in Fig. 1(d) [22], the optical responses of MCNTs mostly originate from $E_{11}$ transitions, and radiative recombination and energy relaxation within the Dirac bands can hardly be observed. Therefore, the dominant relaxation pathway observed in transient transmittance is attributed to the relaxation from the $E_{11}$ van Hove singularity to the Dirac band. The relaxation pathway from the $E_{11}$ band to the Dirac band is forbidden if the sample has a perfect cylindrical symmetry and the angular momentum around the cylinder is conserved. However, external factors such as the presence of surfactants, defects, or impurities can destroy the ideal radial symmetry, which may partially allow transitions via this forbidden pathway. This assumption enables us to qualitatively evaluate the bias dependence of the relaxation rate of transitions from the $E_{11}$ to Dirac band. The other possibility is the contribution from optical phonon scattering, which will also be discussed in the following [27].

In the impact ionization process, the relaxation rate of each excited state with a wave vector of $k_{1}$ should be written by Fermi's golden rule as [26]

$$
\begin{aligned}
S\left(k_{1}, k_{2}, k_{3}, k_{4}\right)= & \frac{2 \pi}{\hbar}\left|\mathcal{M}\left(\left|k_{1}-k_{2}\right|\right)\right|^{2} \delta_{k_{1}+k_{2}-k_{3}-k_{4}} \\
& \times \delta\left(\varepsilon_{1}+\varepsilon_{2}-\varepsilon_{3}-\varepsilon_{4}\right) .
\end{aligned}
$$

Here, $k_{1}$ and $k_{2}$ are the wave vectors of electrons in the initial states, $k_{3}$ and $k_{4}$ are those in the final states, and $\varepsilon_{1}, \varepsilon_{2}, \varepsilon_{3}$, and $\varepsilon_{4}$ are the energies of the corresponding electrons. $\mathcal{M}\left(\left|k_{1}-k_{2}\right|\right)$ is a matrix element of the carrier-carrier scattering due to the screened Coulomb interaction, which we assumed to follow a Bessel polynomial $K_{0}(x)$ in the one-dimensional MCNT by considering the screening effect [28]. We used

$$
\mathcal{M}(q)=\frac{\mathcal{M}_{0}}{L} \frac{K_{0}(q d)}{1+a K_{0}(q d)}
$$

in the calculation. Here, $L$ is the normalization length for a plane wave in one dimension [29]. The parameter $a=$ $8 \alpha c / \pi v_{F} \sim 0.64$, where $v_{F} \sim 343 / c$ is the Fermi velocity, $\alpha \sim 1 / 137$ is the fine structure constant, and $c$ is the speed of light [28]. Two delta functions express the conservation of energy and momentum. Then the scattering rate of the carriers at $k_{1}$ can be calculated by integrating all possible pairs of initial and final states. For example, we calculated the relaxation time of the carriers due to AAB process depicted in Fig. 4(a) (where $A$ and $B$ correspond to the indices of Dirac bands with positive and negative slope in the dispersion near the Dirac point, respectively) using

$$
\begin{aligned}
\tau_{\mathrm{AAB}}^{-1}\left(k_{1}\right)= & \frac{L^{2}}{(2 \pi)^{2}} \iint d k_{2} d k_{3} f\left(k_{2}\right)\left[1-f\left(k_{3}\right)\right] \\
& \times\left[1-f\left(k_{1}+k_{2}-k_{3}\right)\right] \\
& \times S\left(k_{1}, k_{2}, k_{3}, k_{1}+k_{2}-k_{3}\right) .
\end{aligned}
$$

The change in chemical potential (Fermi energy) and temperature was introduced through the Fermi distribution functions $f(k)$. Here, we assumed that the Dirac electrons were at $300 \mathrm{~K}$. Finally, we summed up all possible contributions shown in Fig. 4(a), and integrated as a function of $k_{1}$ assuming that the excited-state population can be described by the 
(a)
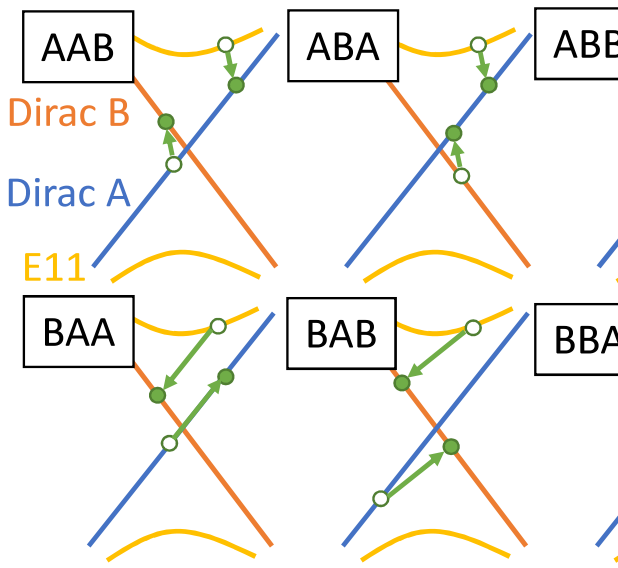

BBA

(b)

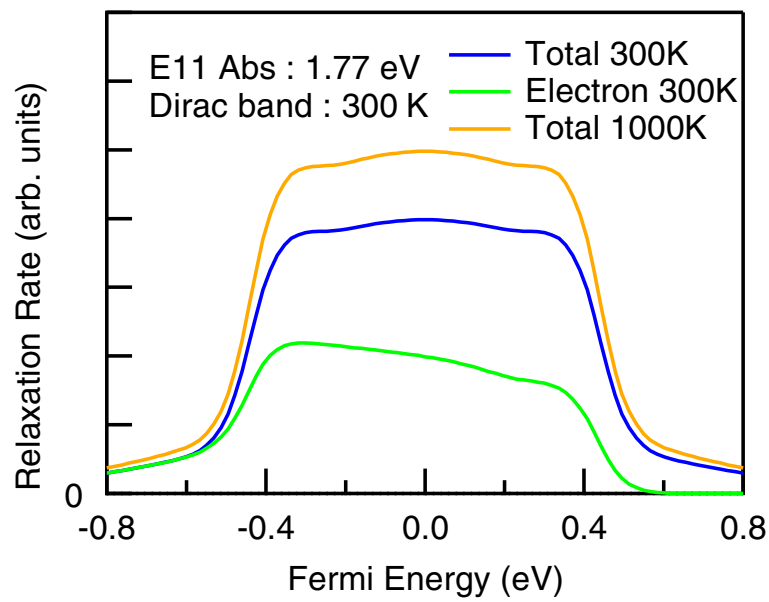

FIG. 4. (a) Possible relaxation pathways of the impact ionization processes for excited carriers in the $E_{11}$ band. (b) Calculated Fermienergy dependence of the relaxation rate of photoexcited carriers. The initial electron distribution of the Dirac band was assumed to be in thermal equilibrium at $300 \mathrm{~K}$. The results of the calculation when the excited electrons in the $E_{11}$ band were at 300 and $1000 \mathrm{~K}$ are shown. Note that we summed the electron and hole relaxation rates as both processes contributed to the bleaching of the MCNT absorption. The Fermi-energy dependence of the electron relaxation rate calculated at $300 \mathrm{~K}$ is also shown.

Boltzmann function $B\left(k_{1}\right) \propto \exp \left(-\varepsilon_{1} / k T_{e}\right)$ with the excitedstate electronic temperature $T_{e}$. Obtained total relaxation rate due to the impact ionization process becomes as follows:

$$
\begin{aligned}
\tau_{I I}^{-1}= & \frac{1}{n} \int d k_{1} B\left(k_{1}\right)\left[\tau_{\mathrm{AAB}}^{-1}\left(k_{1}\right)+\tau_{\mathrm{ABA}}^{-1}\left(k_{1}\right)+\tau_{\mathrm{ABB}}^{-1}\left(k_{1}\right)\right. \\
& \left.+\tau_{\mathrm{BAA}}^{-1}\left(k_{1}\right)+\tau_{\mathrm{BAB}}^{-1}\left(k_{1}\right)+\tau_{\mathrm{BBA}}^{-1}\left(k_{1}\right)\right],
\end{aligned}
$$

where $n=\int d k_{1} B\left(k_{1}\right)$ is the population of excited states.

Figure 4(b) displays the calculated Fermi-energy dependence of the decay rate of photoexcited carriers. To include both electron and hole relaxations, we summed the calculated result for electrons shown in Fig. 4(b) with that for holes (which could be obtained by flipping the energy axis because of the electron-hole symmetry). The total carrier decay rate was at its maximum near the Dirac point, and was almost flat until the Fermi energy reached one-quarter of $E_{11}$ energy $(\sim \pm 0.4 \mathrm{eV})$. The calculated result qualitatively agrees with

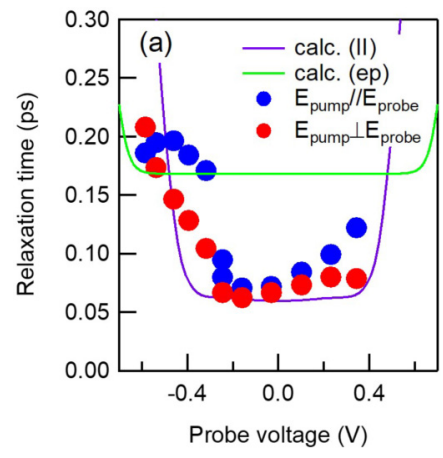

(b)

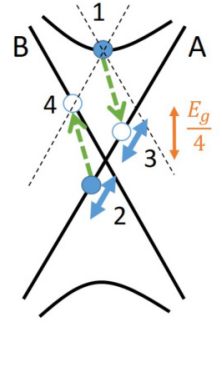

(c)

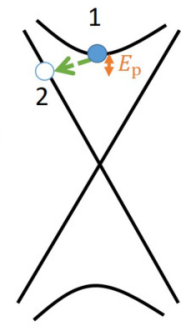

FIG. 5. (a) Comparison of the calculated and experimental relaxation times as a function of the probe voltage. Calculated results of impact ionization (II) and phonon scattering (ep) are plotted as a function of Fermi energy (i.e., probe voltage). (b) Schematic of the relaxation process from the bottom of the $E_{11}$ band due to impact ionization. The calculation for impact ionization process was carried out by integrating the electronic states of 2 and 3, while the electronic state of 4 was fixed at Eg/4 due to energy-momentum conservation. (c) Schematic of the relaxation process due to electron-phonon scattering.

the experimental results shown in Fig. 5(a), given that the Dirac point in this sample was increased to $1.0 \mathrm{~V}$ due to unintentional doping and then the net bias voltage at the sample position was reduced as observed in Fig. 1(b). These results indicate that the large scope for controlling the carrier relaxation rate in MCNTs originates from switching on and off the interband pathways.

Using the similar formalism, we also simulated the relaxation rate due to the electron-phonon scatterings. For simplicity, we assumed that the electron-phonon coupling constant $C$ is independent of the electron wave vector, and the electron-phonon scattering probability was mainly determined by the distribution functions of Dirac and band electrons. The relaxation rate due to the phonon scattering process could be written as

$$
\tau_{\mathrm{ep}}^{-1} \propto \int d k_{1} C B\left(k_{1}\right)\left[1-f\left(k_{2}\right)\right] .
$$

Here, $k_{1}$ and $k_{2}$ represent the wave vectors of the initial and final states for electron-phonon scattering. The wave vector of the final state could be calculated from the relation $\varepsilon_{1}\left(k_{1}\right)-\varepsilon_{2}\left(k_{2}\right)=\varepsilon_{p}$, where $\varepsilon_{p}$ is the phonon energy. The calculated relaxation time due to the electron-phonon interaction is plotted by the green curve in Fig. 5(a). [Note that the absolute value of the relaxation time could not be obtained from Eq. (5), and the position of the green curve is arbitrary.] The calculated result exhibits the presage of Pauli blocking at higher bias voltage than the experimental result, indicating that the impact ionization process mainly contributes to the carrier relaxation process at low Fermi energies. On the other hand, the deviation from the impact ionization model observed at the higher probe voltage $(|V|>0.4 \mathrm{~V})$ might come from the contribution of the electron-phonon scattering process, which is expected to last until the Fermi energy reaches higher bias voltage comparable to the $E_{11}$.

The reason why the relaxation time for impact ionization process changes at one-quarter of that of the $E_{11}$ transition 
could be understood as follows. If we consider the relaxation from the minimum of the $E_{11}$ band through the AAB process, as shown by the electronic state 1 in Fig. 5(b), the energy and momentum conservation result in a fixed final state [the electronic state 4 in Fig. 5(b)]; the integration was performed over the $A$ band while keeping the distance between states 2 and 3 constant. Because the energy of electronic state 4 was one-quarter of that of the $E_{11}$ transition, and the main pathway for the electronic relaxation originated from the energy minimum of the $E_{11}$ band, the relaxation rate changed drastically when the Fermi energy reached one-quarter of that of the $E_{11}$ transition. Hence, the entire AAB process was blocked.

Impact ionization has been both theoretically and experimentally investigated, mainly in graphene [26,30]. The time scale of the intraband carrier relaxation in graphene is faster than $100 \mathrm{fs}$ and that of the interband seems to be slower [3,31]. The observed relaxation time in MCNTs is between these two contributions, which may originate from the 1D characteristic of MCNTs that makes the electron-electron scattering more frequent than in graphene. The radial symmetry breaking in MCNTs assumed here is also required for the existence of long-range Coulomb interactions. These points could be further elucidated by measuring the carrier dynamics while changing the gate voltage of suspended MCNTs and graphene, as environmental effects would be reduced under such conditions. However, these results demonstrate that the relaxation pathways for the carriers could effectively be modulated via the gate bias voltage, which could be important for future devices using carbon-based nanomaterials.

\section{CONCLUSION}

In conclusion, we observed a strong bias dependence of the carrier relaxation time in 1.4-nm-diameter MCNTs by electrochemical doping of the carriers through ionic liquid. The observed behavior could be understood by 1D impact ionization processes that change the lifetime due to Pauli blocking. Using a theoretical model, we qualitatively reproduced the bias dependence of the relaxation rate. Because of the high controllability of the excited-state relaxation times in the MCNTs, they may have promising applications in future optoelectronics technology.

\section{ACKNOWLEDGMENTS}

We acknowledge Dr. Ken-ichi Sasaki at NTT Basic Research Laboratories for fruitful discussion. This work was partly supported by a Grant-in-Aid for Scientific Research (KAKENHI Grants No. 26107517, No. 17H06124, No. 16H03820, No. 16H06364, No. 16H04001, and No. 16H00919). K.Y. acknowledges support by JST CREST Grant No. JPMJCR17I5. We also thank Naoko Kondo for her support in fabricating the MCNT film samples.
[1] N. M. Gabor, Z. Zhong, K. Bosnick, J. Park, and P. L. McEuen, Science 325, 1367 (2009).

[2] Y. Kawano, T. Fuse, S. Toyokawa, T. Uchida, and K. Ishibashi, J. Appl. Phys. 103, 034307 (2008).

[3] S. Winnerl, M. Orlita, P. Plochocka, P. Kossacki, M. Potemski, T. Winzer, E. Malic, A. Knorr, M. Sprinkle, C. Berger, W. A. de Heer, H. Schneider, and M. Helm, Phys. Rev. Lett. 107, 237401 (2011).

[4] S. Y. Set, H. Yaguchi, Y. Tanaka, and M. Jablonski, J. Lightwave Technol. 22, 51 (2004).

[5] T. Dürkop, S. A. Getty, E. Cobas, and M. S. Fuhrer, Nano Lett. 4, 35 (2004).

[6] S. Berber, Y.-K. Kwon, and D. Tománek, Phys. Rev. Lett. 84, 4613 (2000).

[7] S. M. Bachilo, M. S. Strano, C. Kittrell, R. H. Hauge, R. E. Smalley, and R. B. Weisman, Science 298, 2361 (2002).

[8] A. Hagen, M. Steiner, M. B. Raschke, C. Lienau, T. Hertel, H. Qian, A. J. Meixner, and A. Hartschuh, Phys. Rev. Lett. 95, 197401 (2005).

[9] T. Hertel and G. Moos, Phys. Rev. Lett. 84, 5002 (2000).

[10] F. Wang, G. Dukovic, L. E. Brus, and T. F. Heinz, Phys. Rev. Lett. 92, 177401 (2004).

[11] S. A. Jensen, R. Ulbricht, A. Narita, X. Feng, K. Mullen, T. Hertel, D. Turchinovich, and M. Bonn, Nano Lett. 13, 5925 (2013).

[12] Y. Z. Ma, J. Stenger, J. Zimmermann, S. M. Bachilo, R. E. Smalley, R. B. Weisman, and G. R. Fleming, J. Chem. Phys. 120, 3368 (2004).
[13] A. Ueda, K. Matsuda, T. Tayagaki, and Y. Kanemitsu, Appl. Phys. Lett. 92, 233105 (2008).

[14] M. S. Arnold, A. A. Green, J. F. Hulvat, S. I. Stupp, and M. C. Hersam, Nat. Nanotechnol. 1, 60 (2006).

[15] T. Igarashi, H. Kawai, K. Yanagi, N. T. Cuong, S. Okada, and T. Pichler, Phys. Rev. Lett. 114, 176807 (2015).

[16] J. C. Johannsen, S. Ulstrup, A. Crepaldi, F. Cilento, M. Zacchigna, J. A. Miwa, C. Cacho, R. T. Chapman, E. Springate, F. Fromm, C. Raidel, T. Seyller, P. D. C. King, F. Parmigiani, M. Grioni, and P. Hofmann, Nano Lett. 15, 326 (2015).

[17] K. Yanagi, R. Moriya, Y. Yomogida, T. Takenobu, Y. Naitoh, T. Ishida, H. Kataura, K. Matsuda, and Y. Maniwa, Adv. Mater. 23, 2811 (2011).

[18] M. Kalbac, A. A. Green, M. C. Hersam, and L. Kavan, ACS Nano 4, 459 (2010).

[19] S. Kazaoui, N. Minami, R. Jacquemin, H. Kataura, and Y. Achiba, Phys. Rev. B 60, 13339 (1999).

[20] J. Ye, M. F. Craciun, M. Koshino, S. Russo, S. Inoue, H. Yuan, H. Shimotani, A. F. Morpurgo, and Y. Iwasa, Proc. Natl. Acad. Sci. USA 108, 13002 (2011).

[21] S. Ghosh, S. M. Bachilo, and R. B. Weisman, Nat. Nanotechnol. 5, 443 (2010).

[22] E. H. Haroz, J. G. Duque, X. Tu, M. Zheng, A. R. Hight Walker, R. H. Hauge, S. K. Doorn, and J. Kono, Nanoscale 5, 1411 (2013).

[23] Q. Zhang, E. H. Haroz, Z. Jin, L. Ren, X. Wang, R. S. Arvidson, A. Luttge, and J. Kono, Nano Lett. 13, 5991 (2013).

[24] Y. Hashimoto, Y. Murakami, S. Maruyama, and J. Kono, Phys. Rev. B 75, 245408 (2007). 
[25] S. Tani, F. Blanchard, and K. Tanaka, Phys. Rev. Lett. 109, 166603 (2012).

[26] A. Girdhar and J. P. Leburton, Appl. Phys. Lett. 99, 043107 (2011).

[27] T. Hertel, V. Perebeinos, J. Crochet, K. Arnold, M. Kappes, and P. Avouris, Nano Lett. 8, 87 (2008).
[28] K. Sasaki, Phys. Rev. B 65, 195412 (2002).

[29] A. Mošková and M. Moško, Phys. Rev. B 49, 7443 (1994).

[30] L. Pirro, A. Girdhar, Y. Leblebici, and J.-P. Leburton, J. Appl. Phys. 112, 093707 (2012).

[31] J. M. Dawlaty, S. Shivaraman, M. Chandrashekhar, F. Rana, and M. G. Spencer, Appl. Phys. Lett. 92, 042116 (2008). 